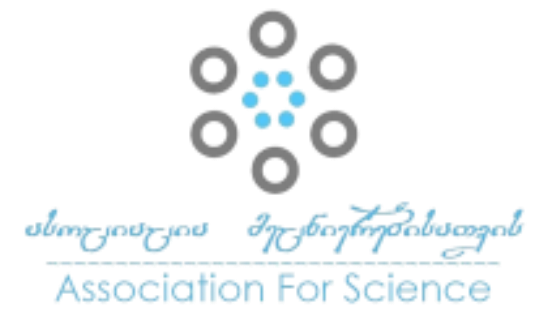

Georgian Scientists

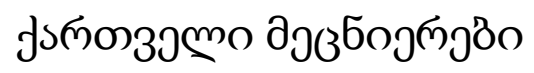

Vol. 4 Issue 1, 2022

https://doi.org/10.52340/gs.2022.04.01.13

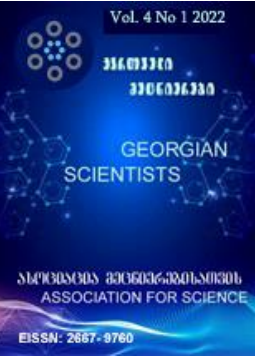

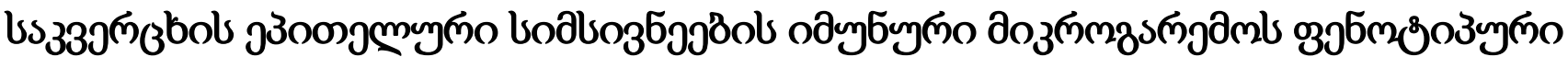

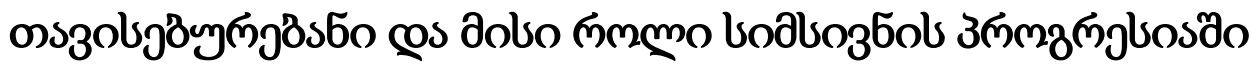

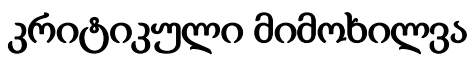

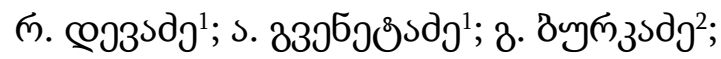

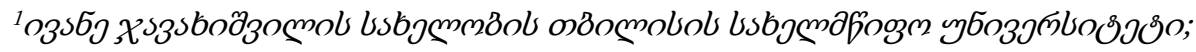

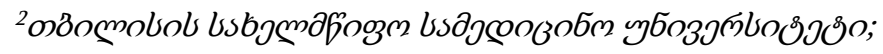

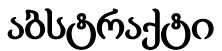

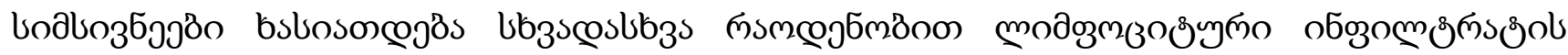

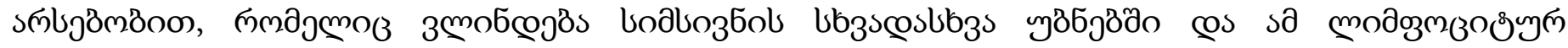

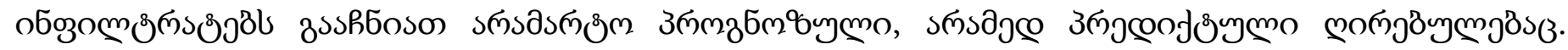

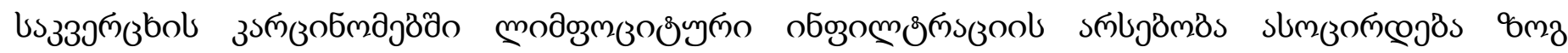

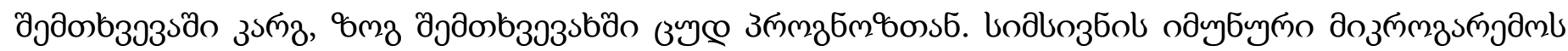

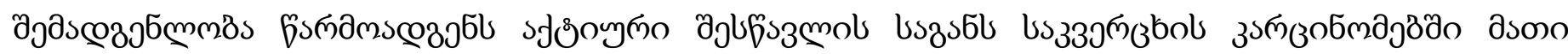

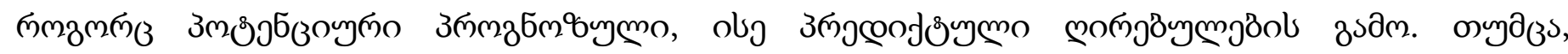

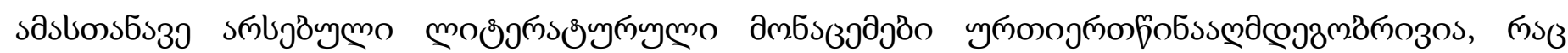

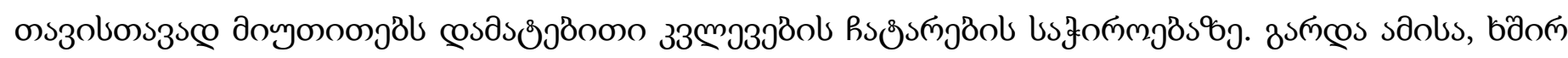

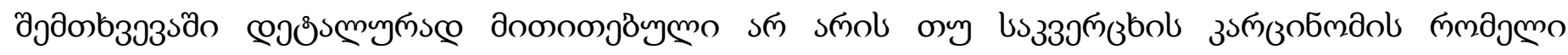

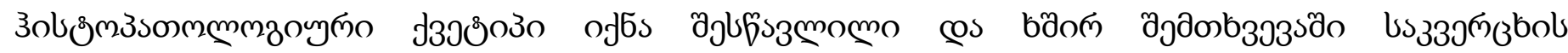

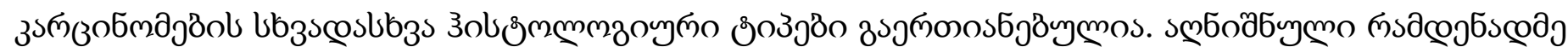

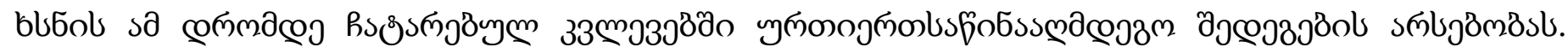

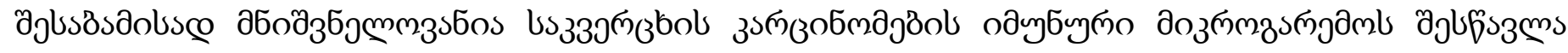

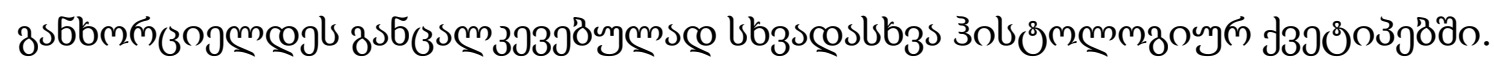

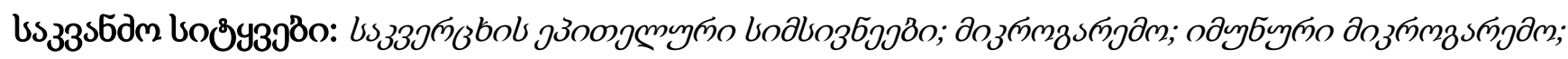




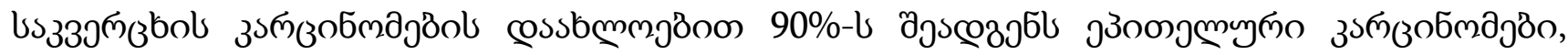

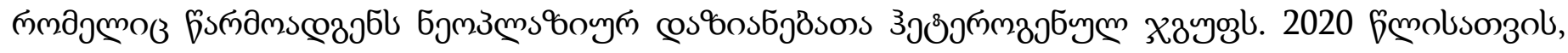

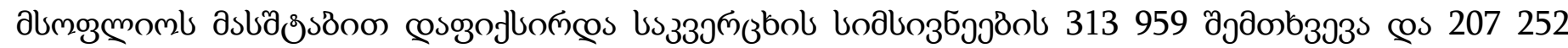

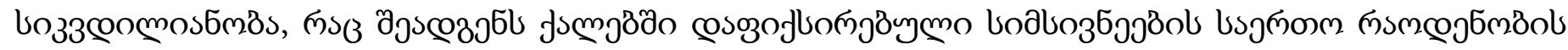

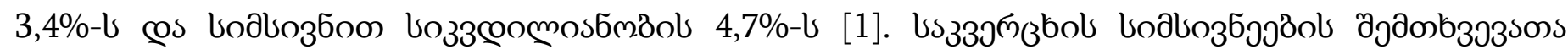

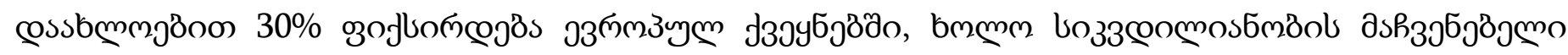

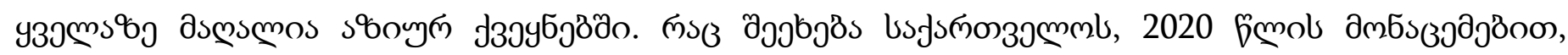

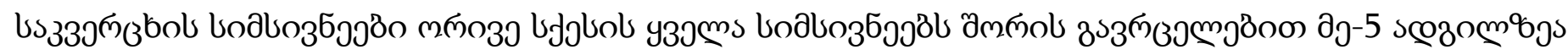

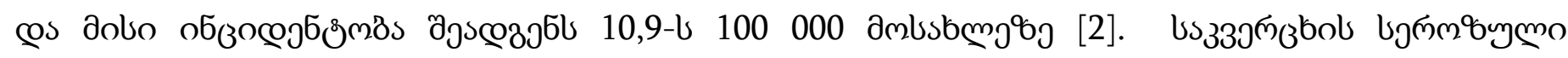

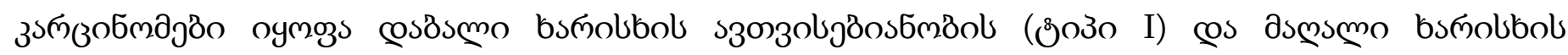

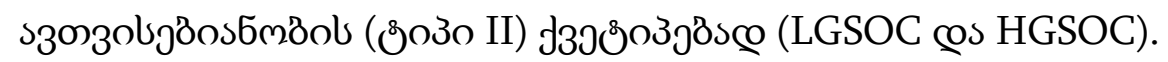

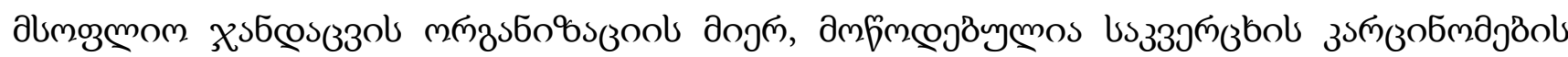

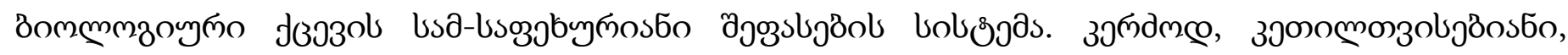

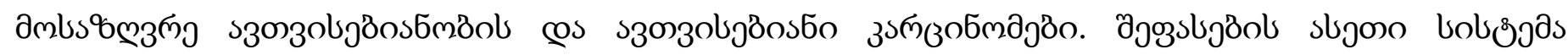

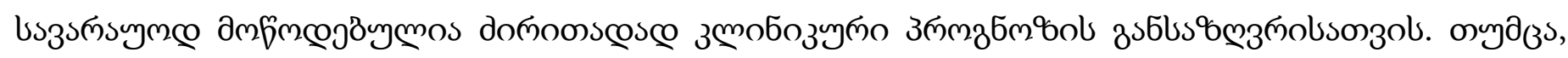

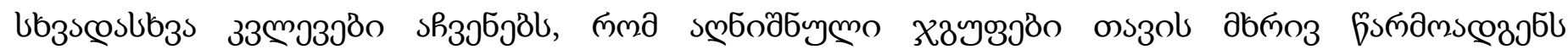

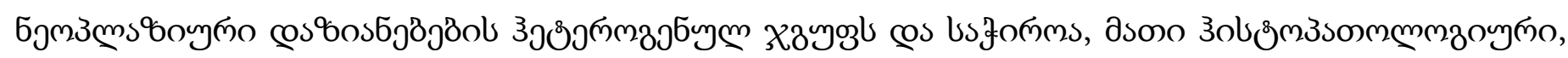

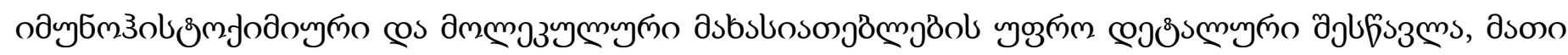

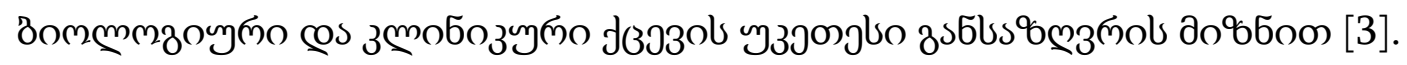

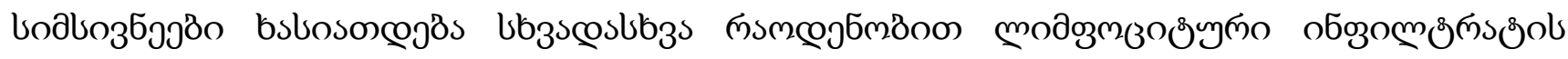

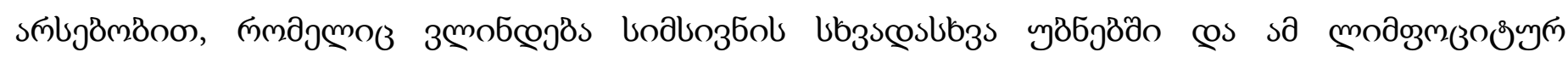

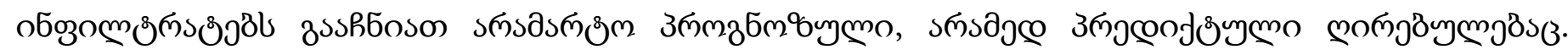

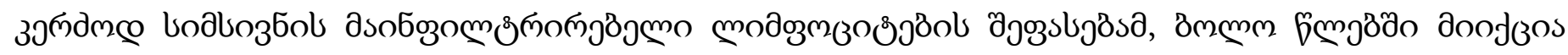

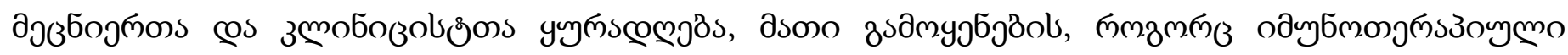

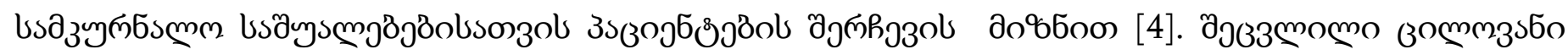

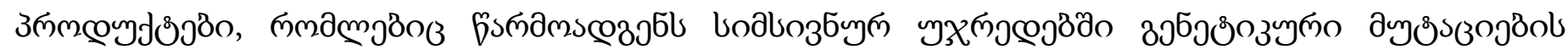

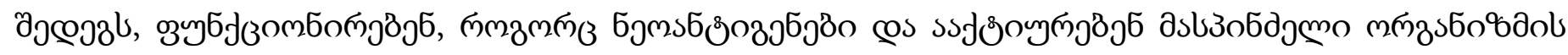

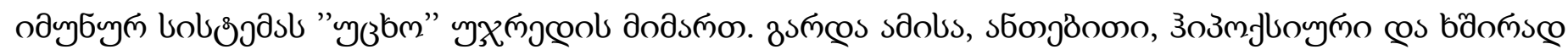

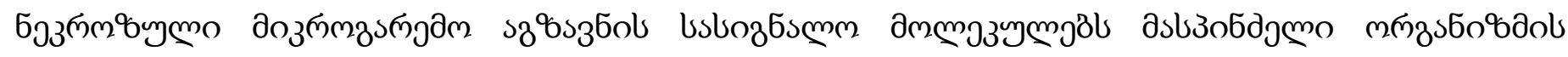

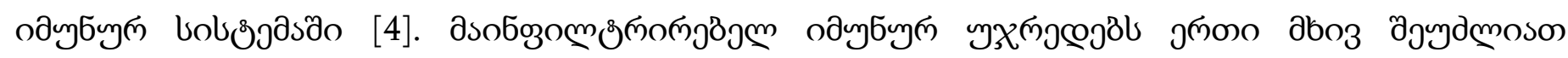

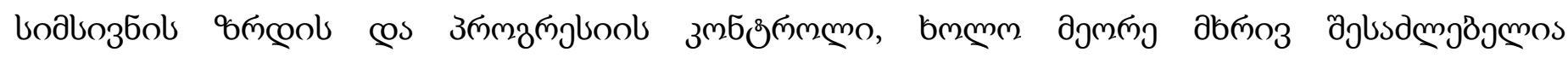

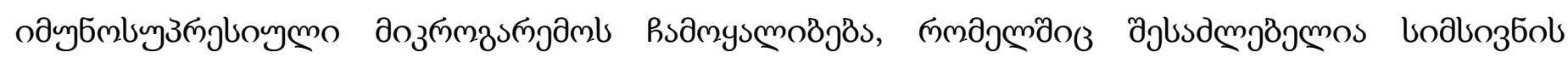

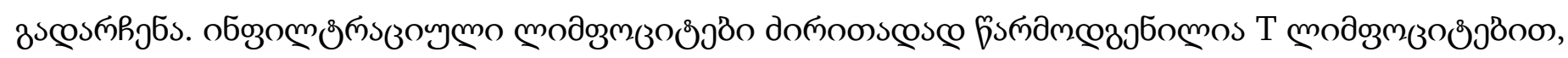

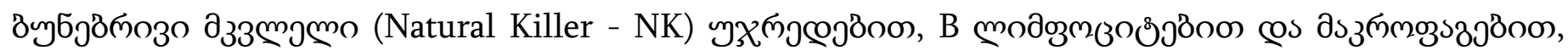

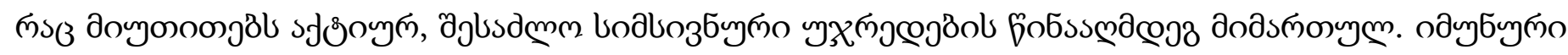

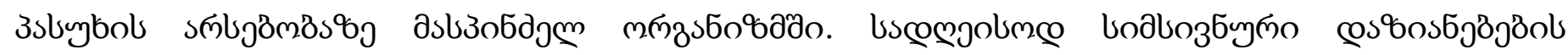




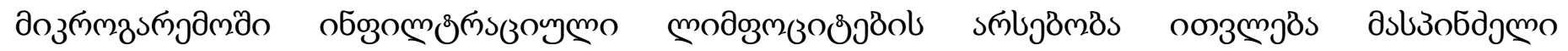

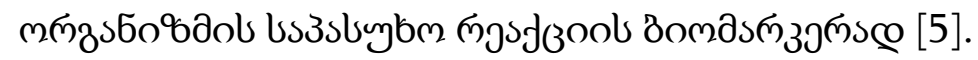

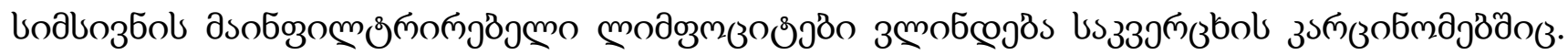

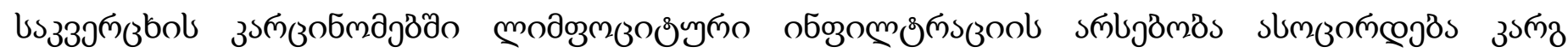

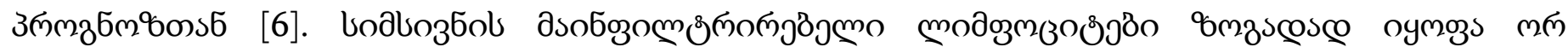

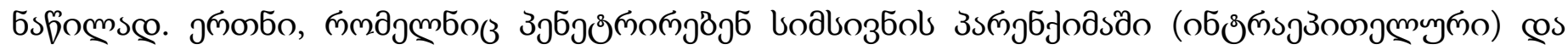

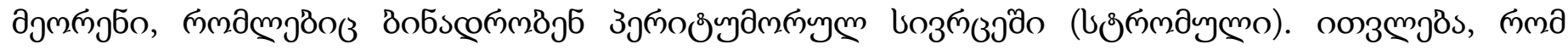

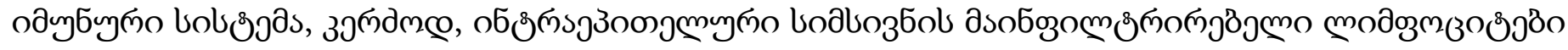

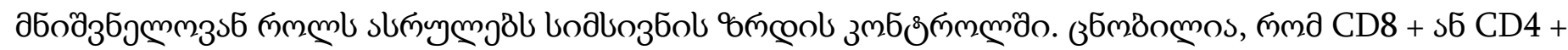
$\mathrm{T}$ модозизољ

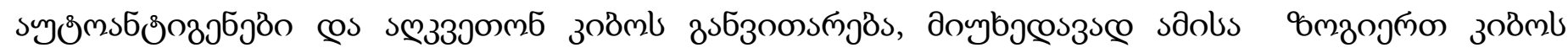

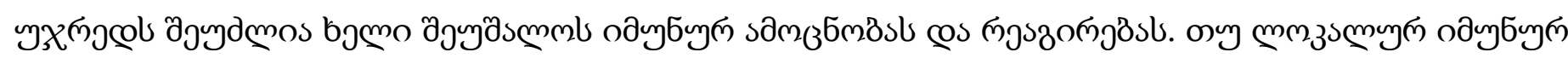

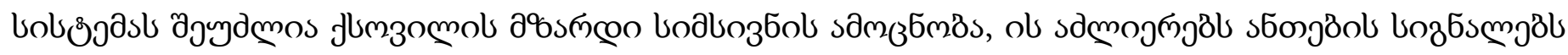

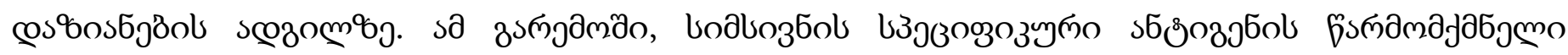

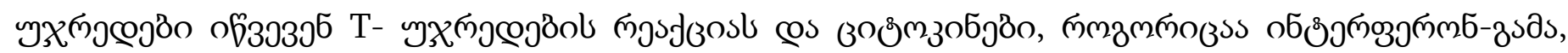

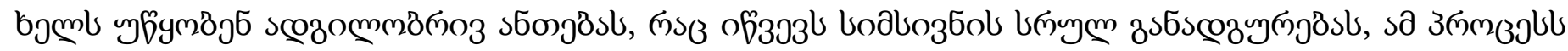

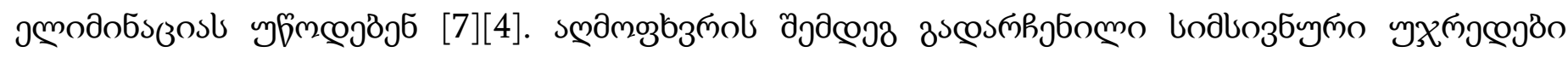

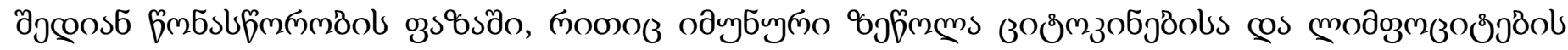

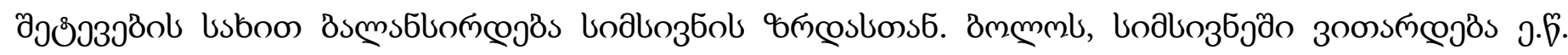

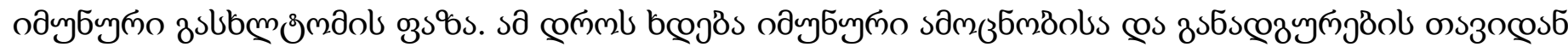

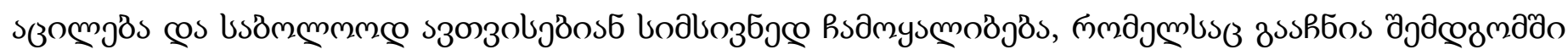

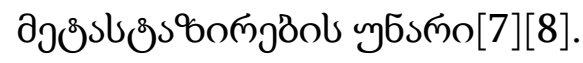

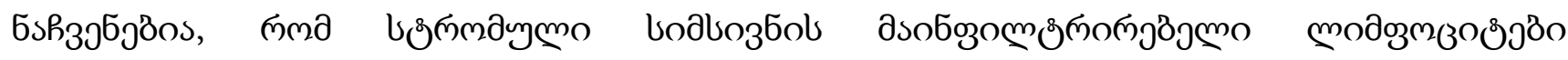

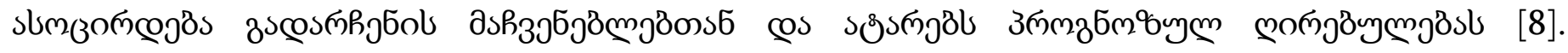

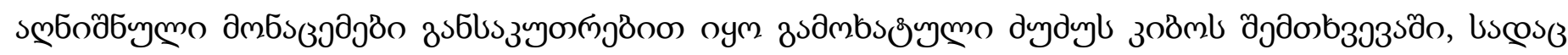

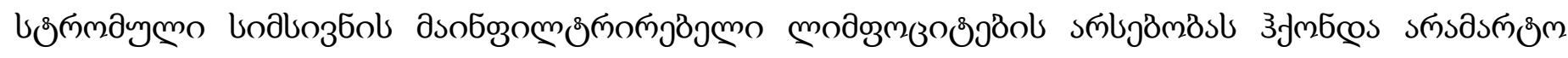

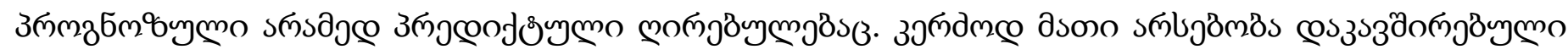

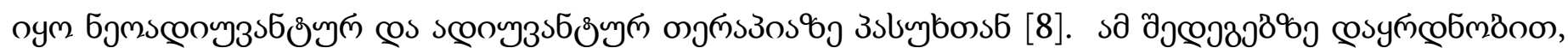

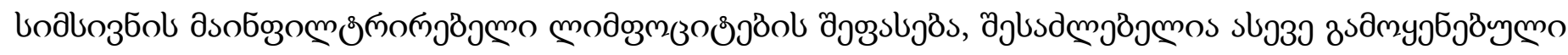

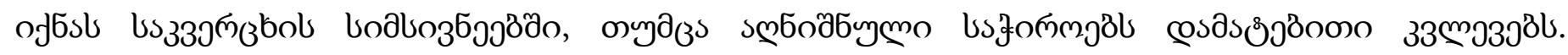

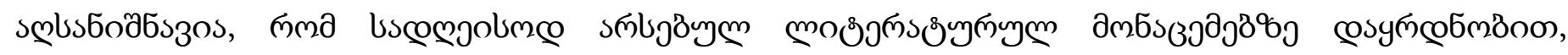

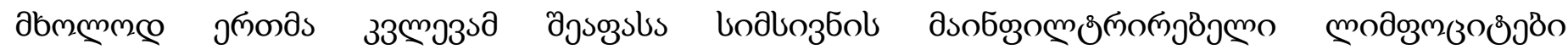

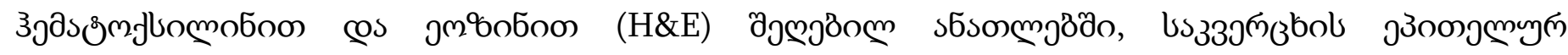

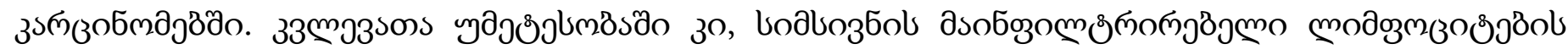

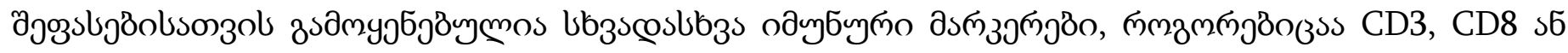

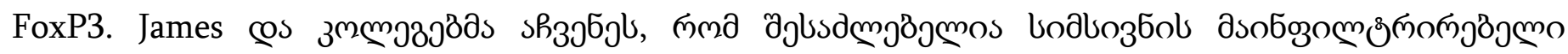




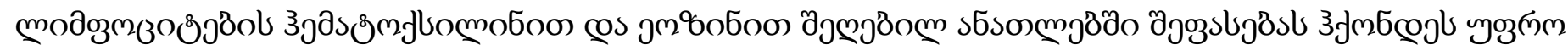

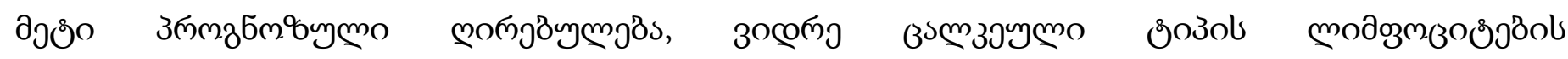

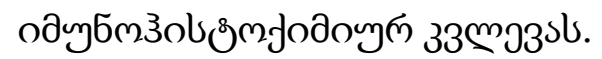

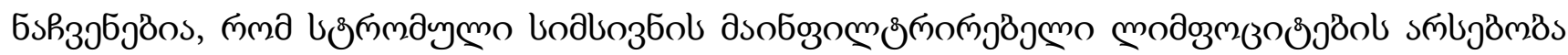

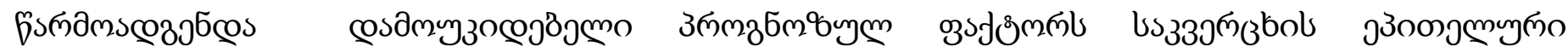

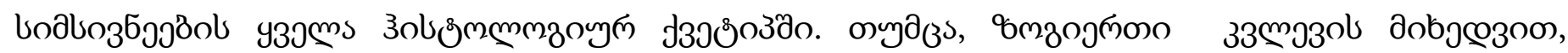

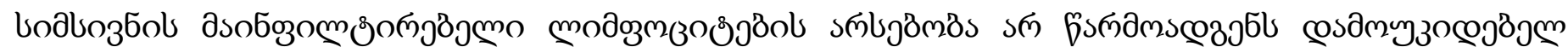

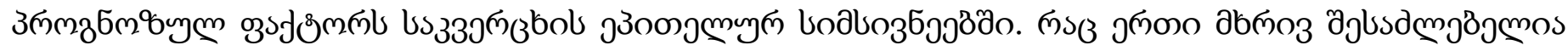

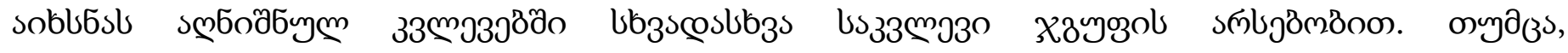

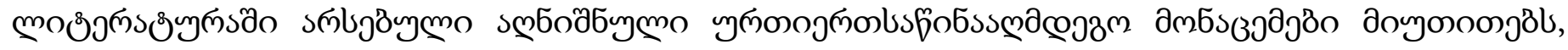

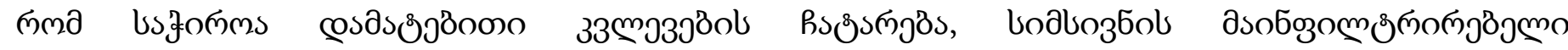

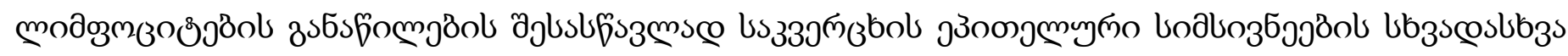

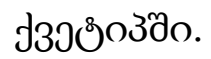

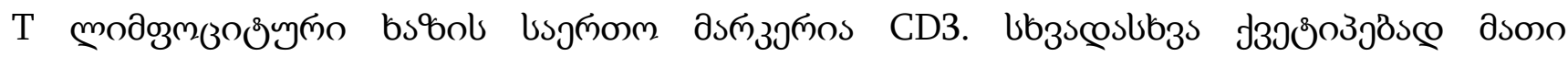

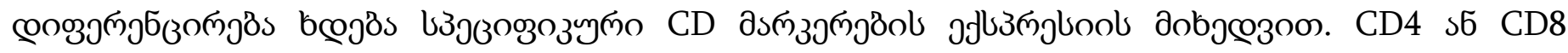

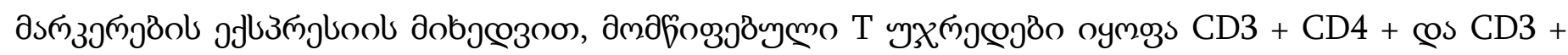

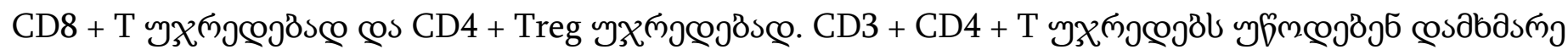

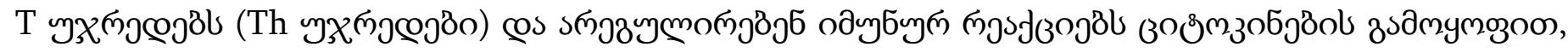

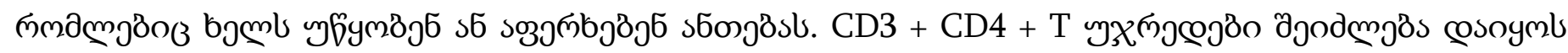

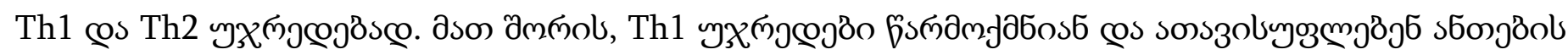

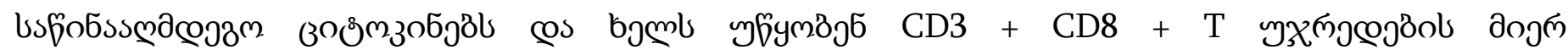

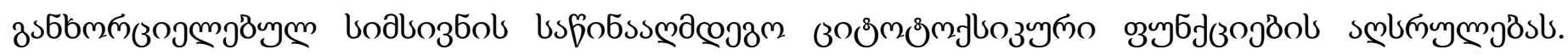

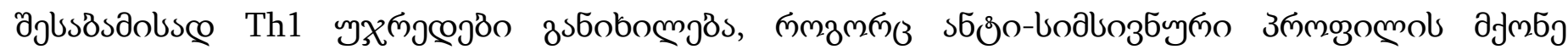

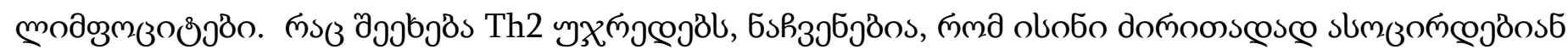

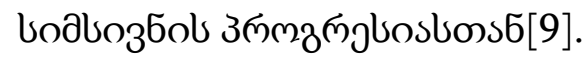

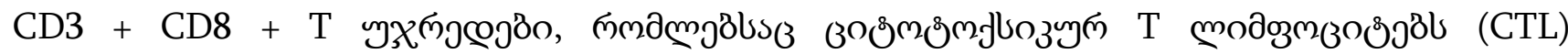

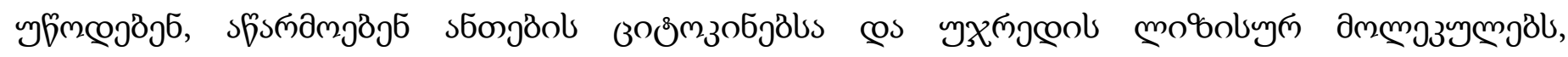

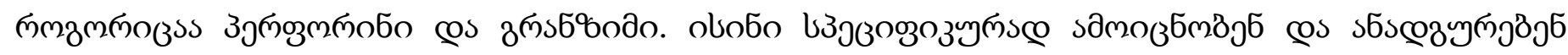

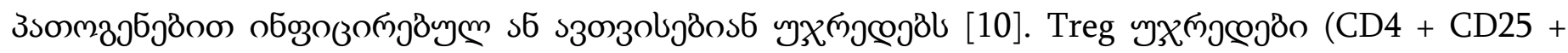

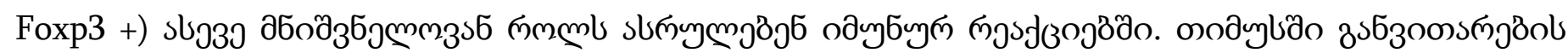

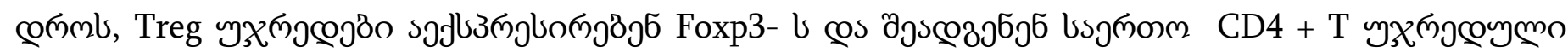

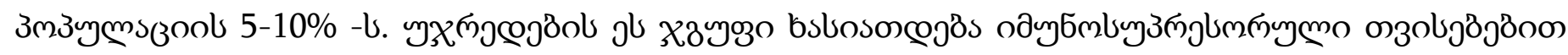

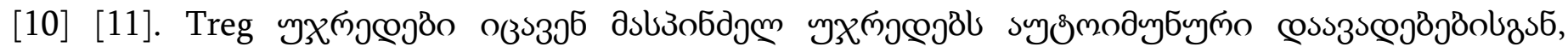

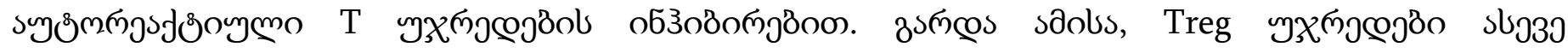

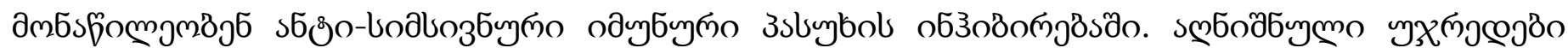

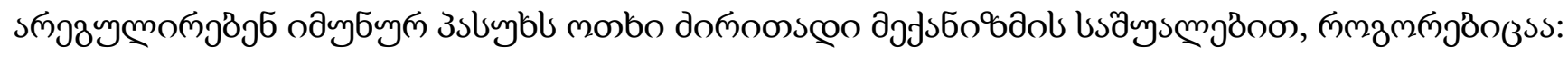




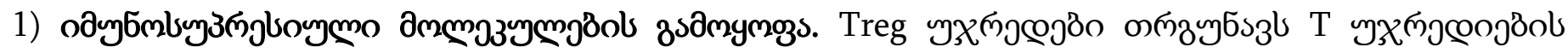

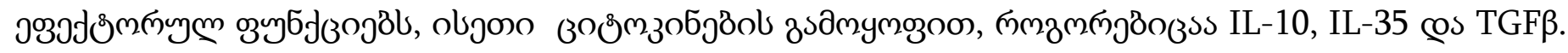

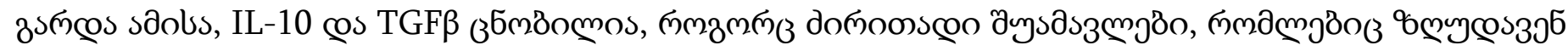

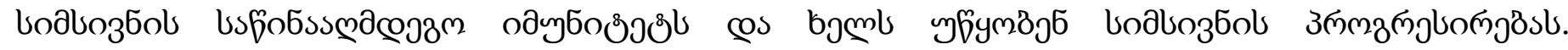
иsобతి

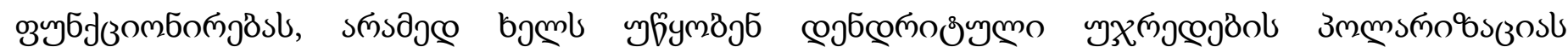

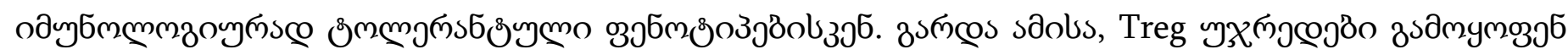

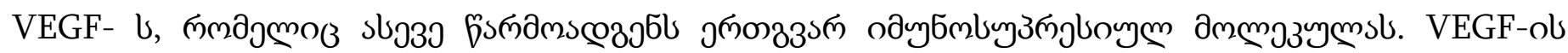

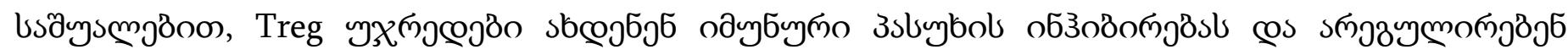

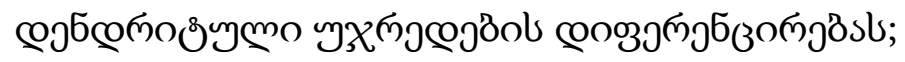

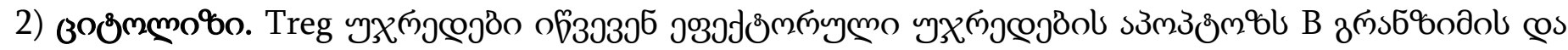

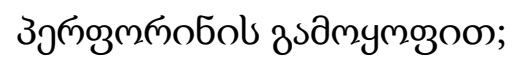

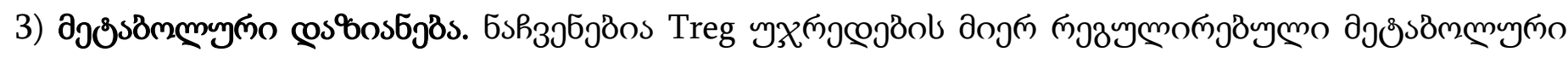

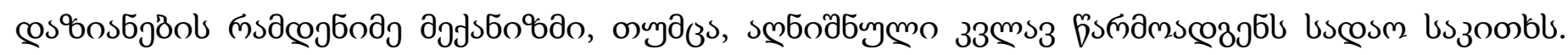

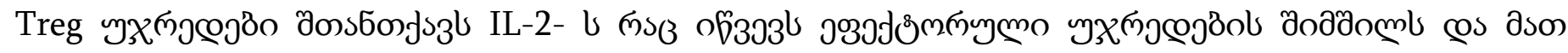

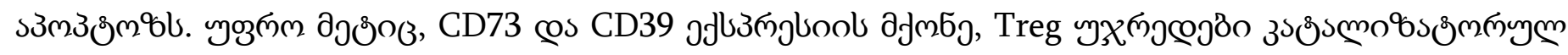

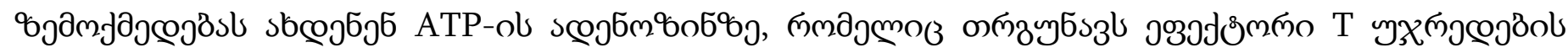

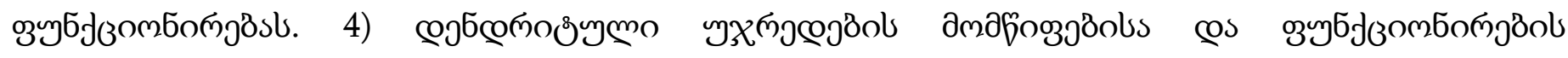

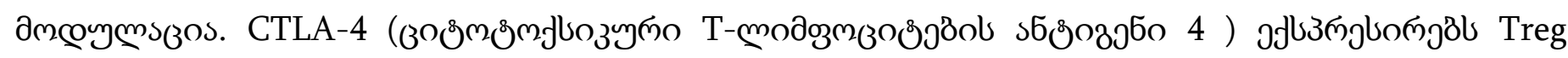

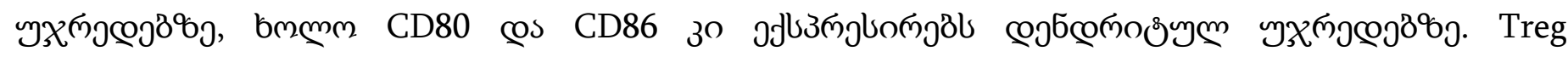

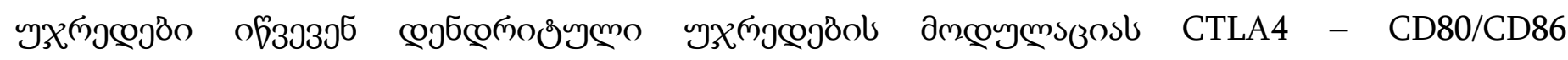

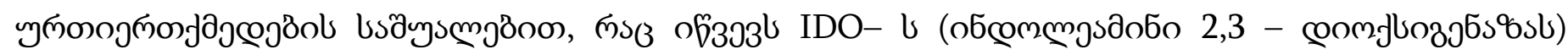

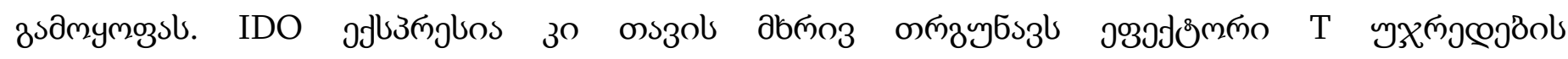

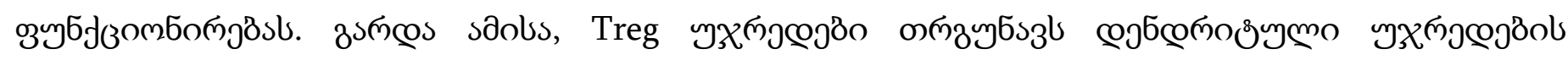

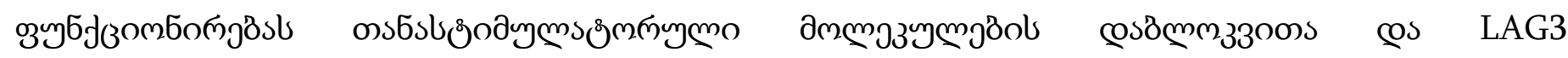

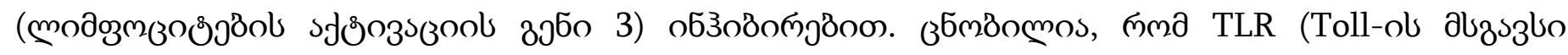

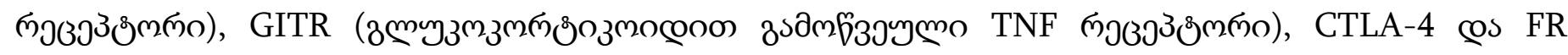

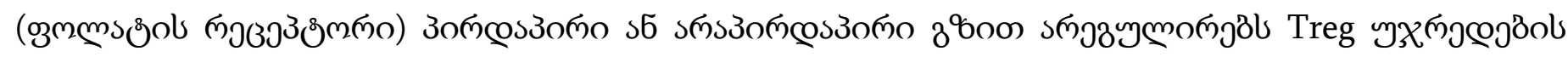

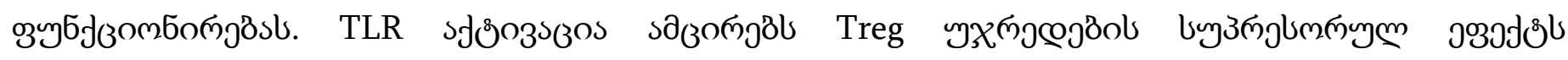

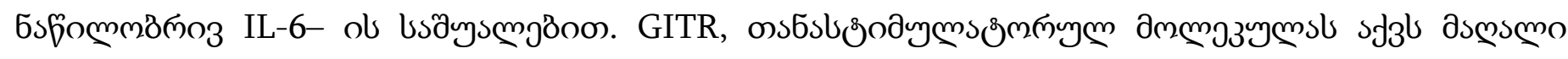

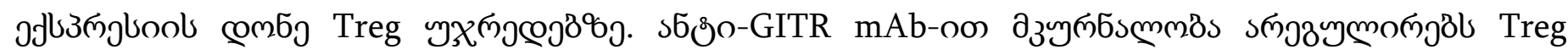

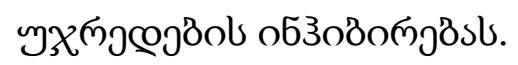

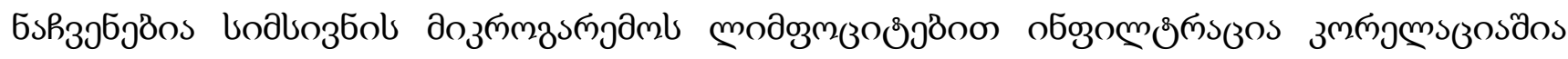

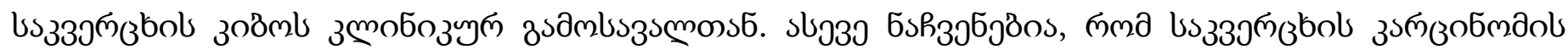

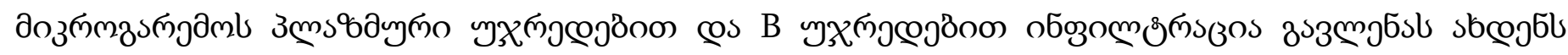

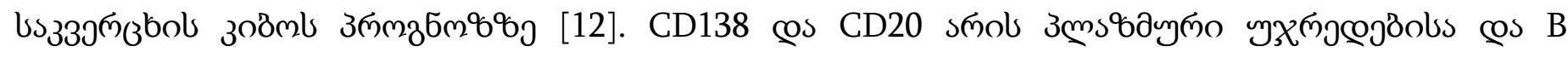

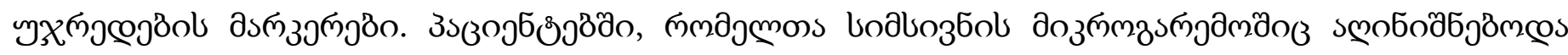




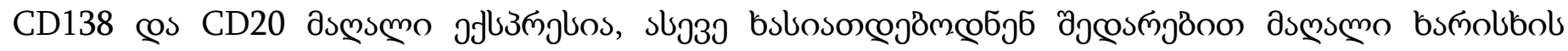

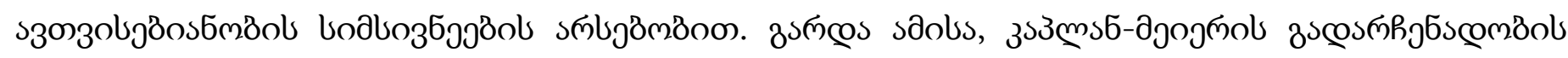

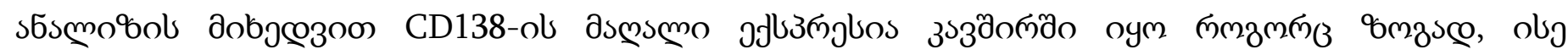

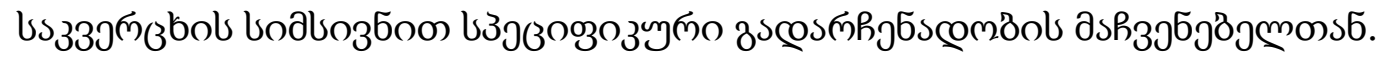

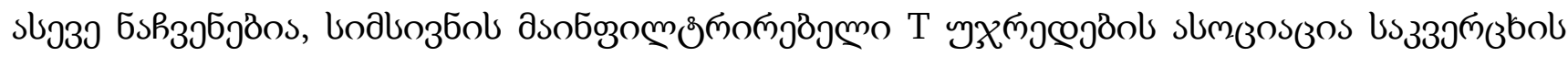

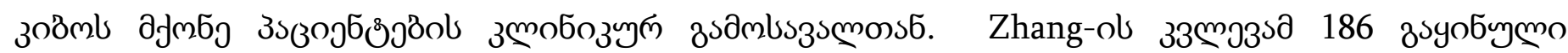

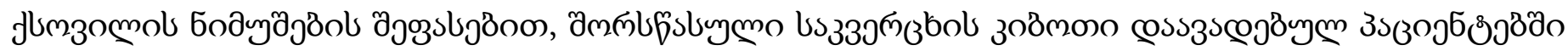

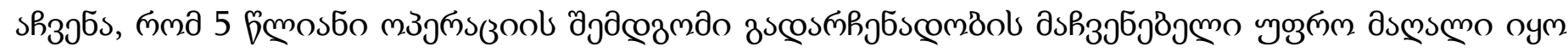

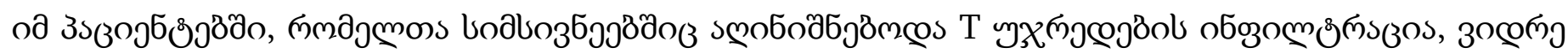

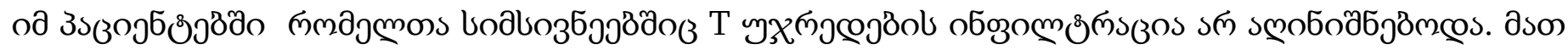

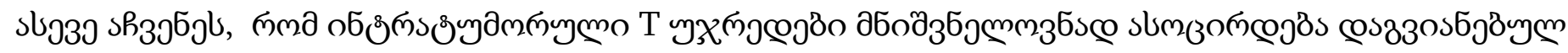
๓эзомозоль [13].

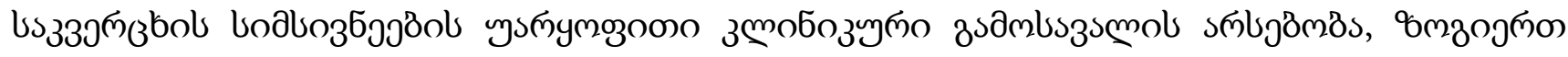

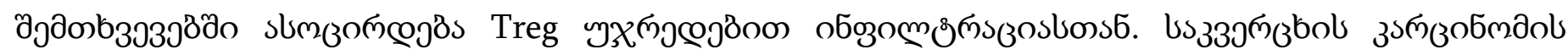

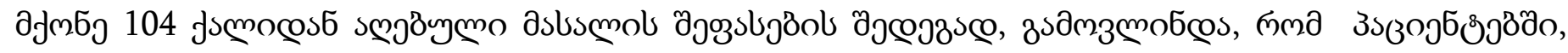

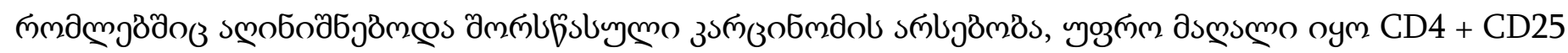

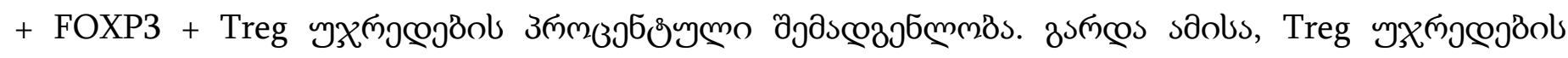

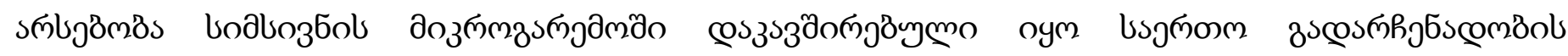

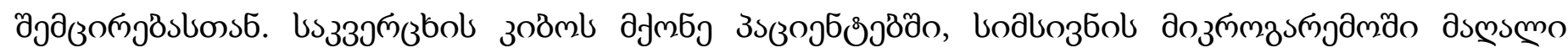

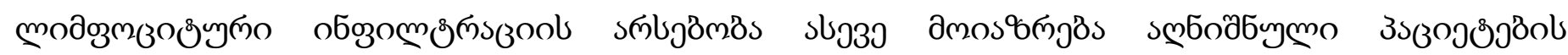

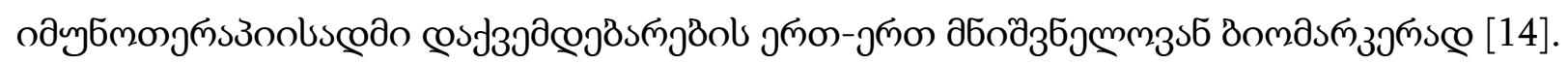

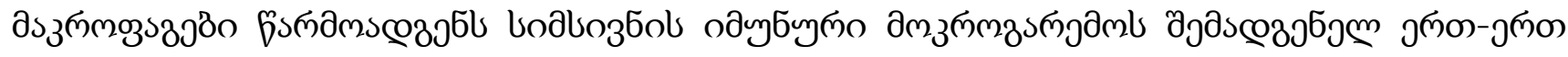

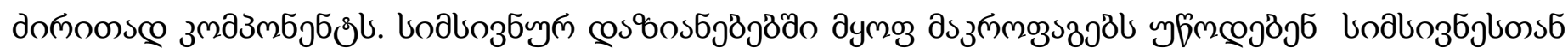

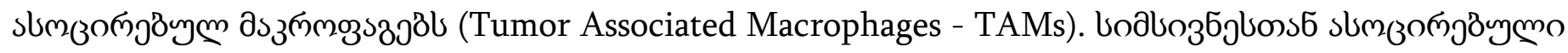

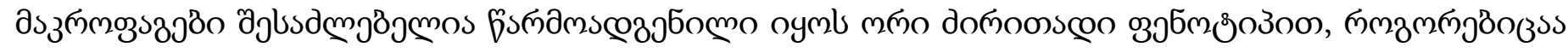

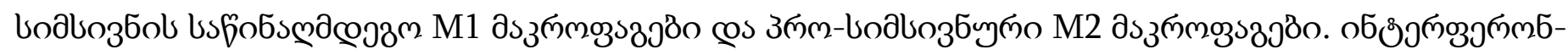

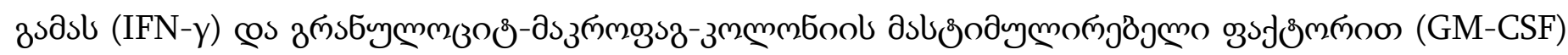

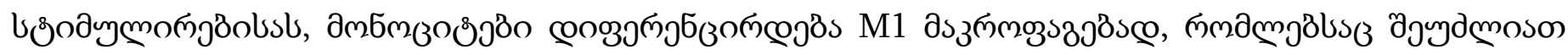

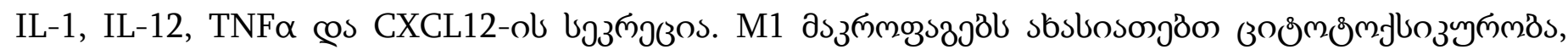

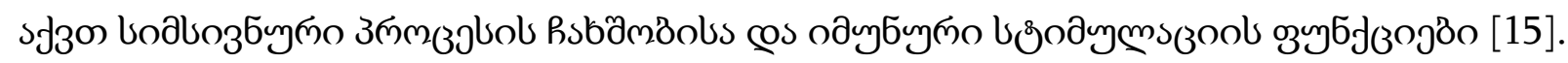

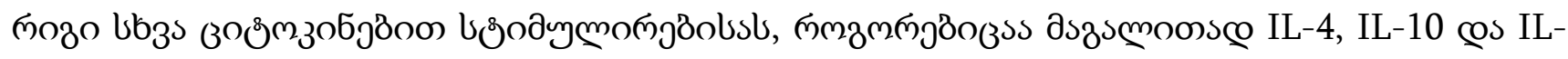

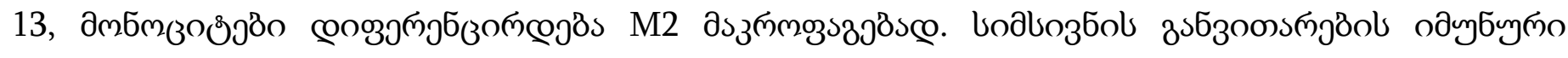

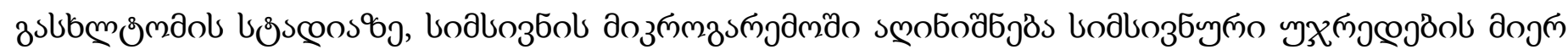

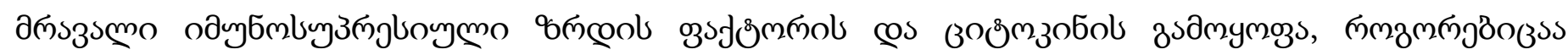

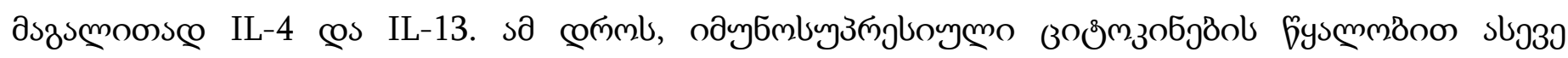




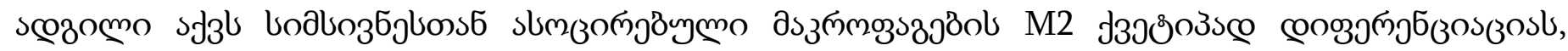

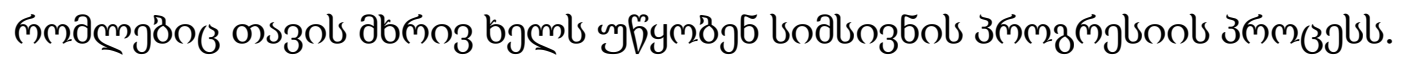

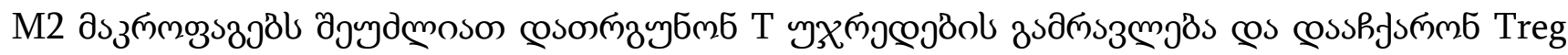

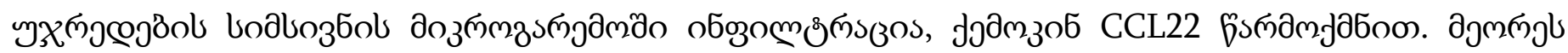

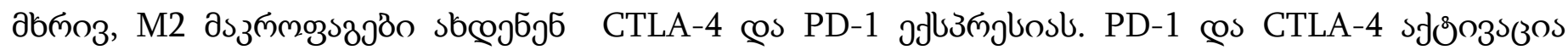

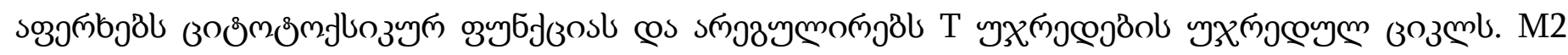

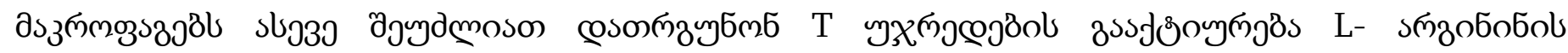

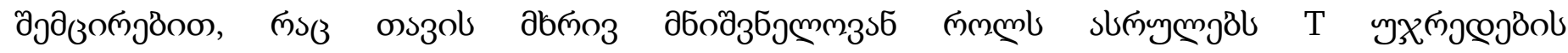

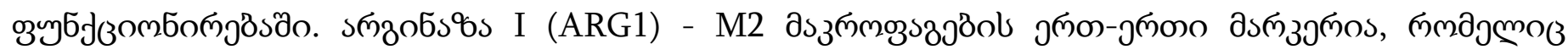

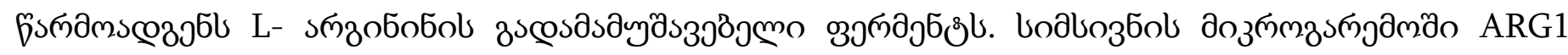

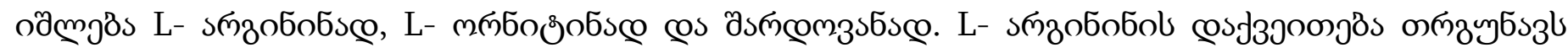

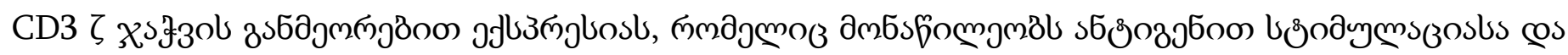

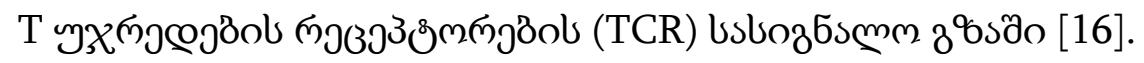

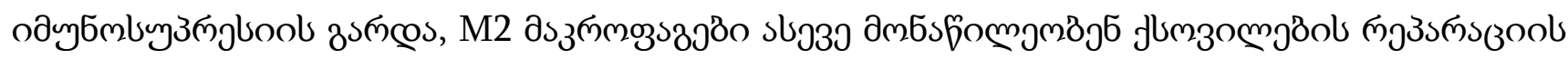

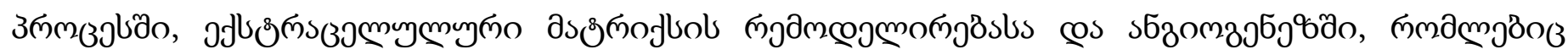

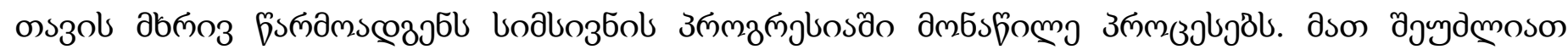

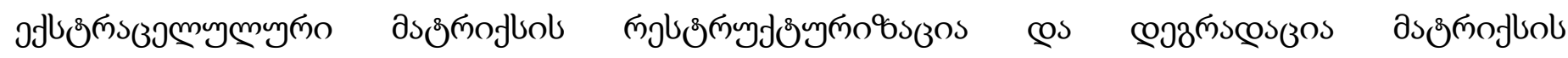

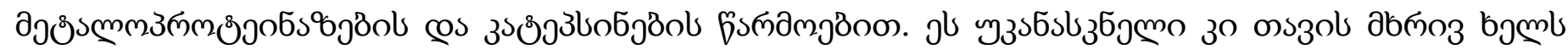

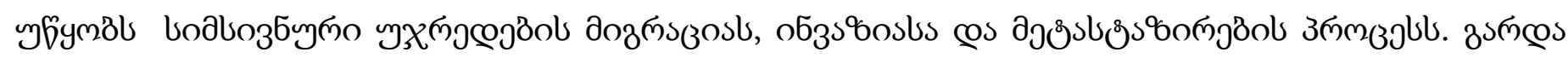

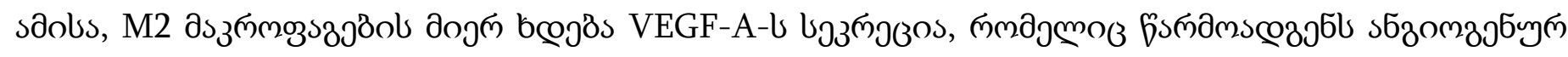

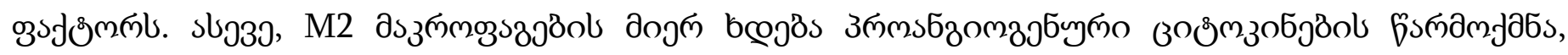

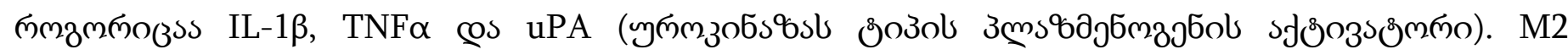

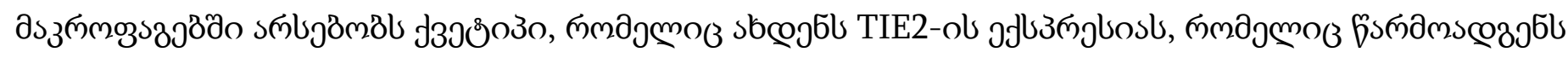

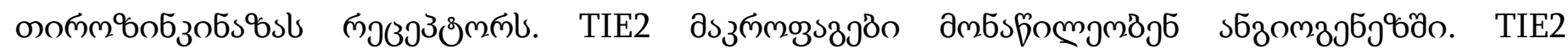

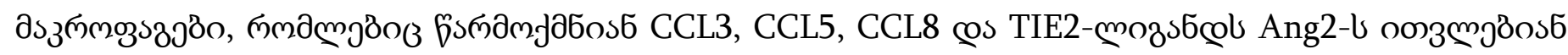

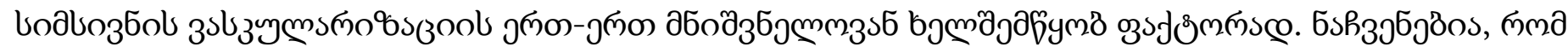

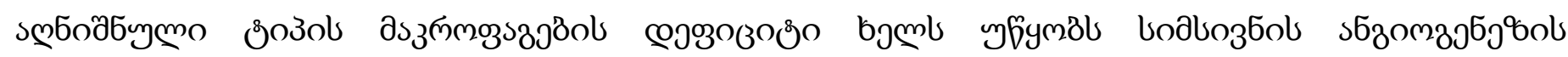

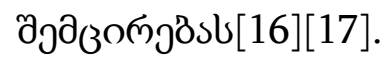

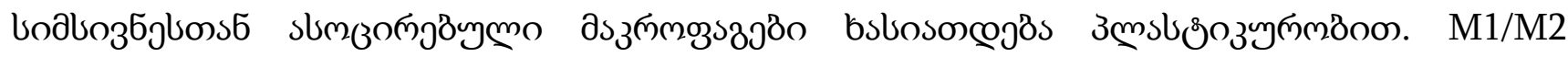

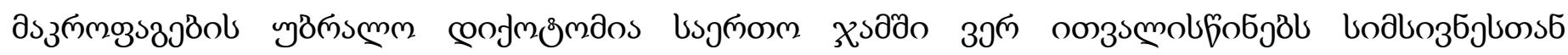

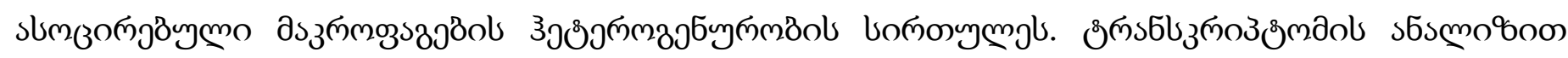

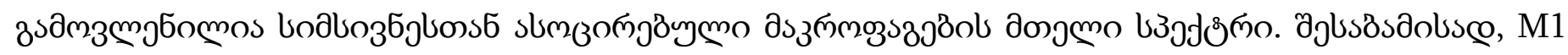

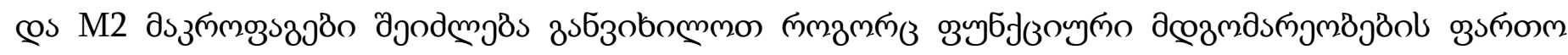

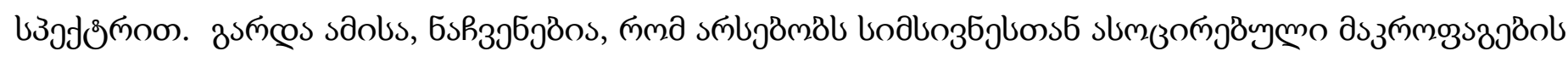

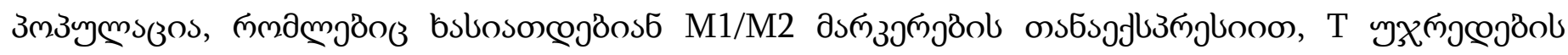

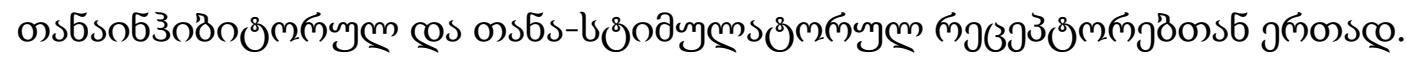




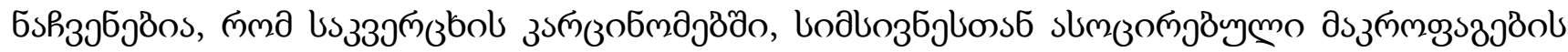

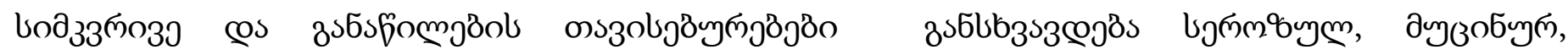

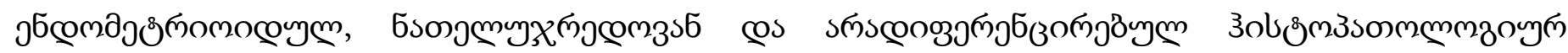

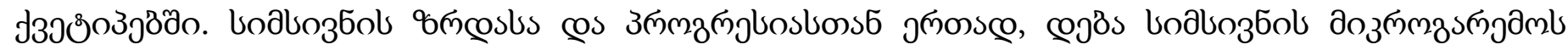

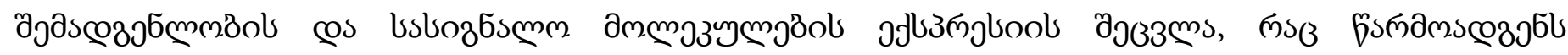

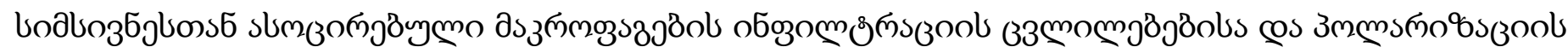

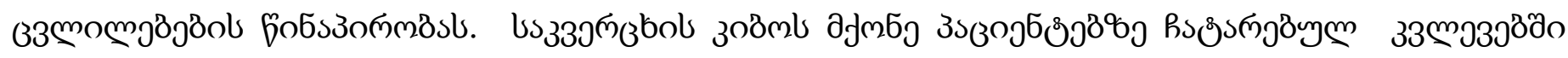

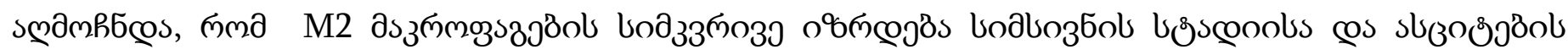

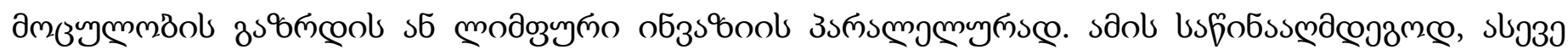

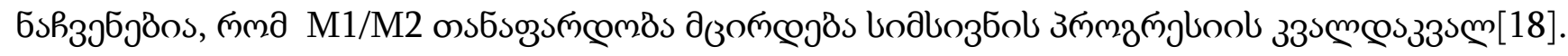

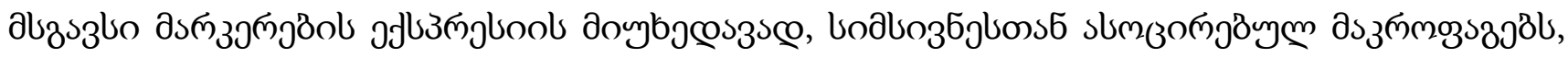

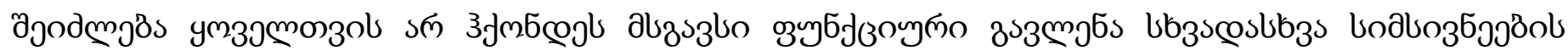

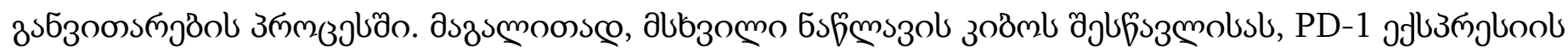

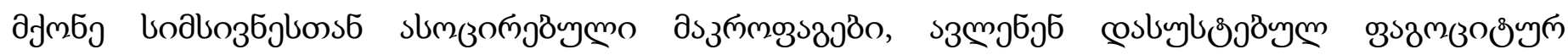

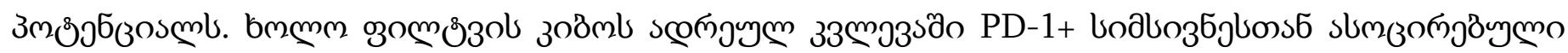

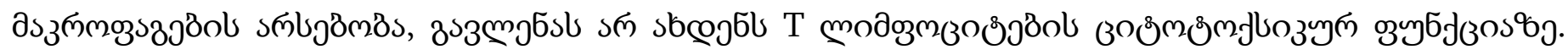

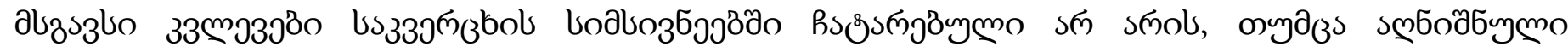

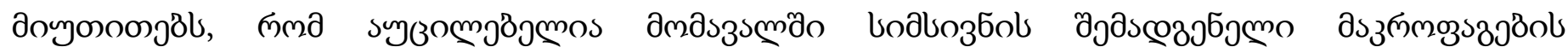

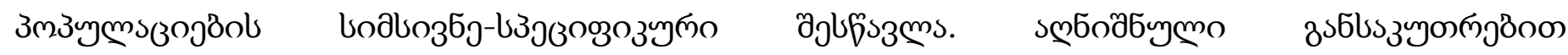

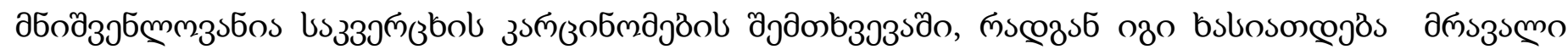

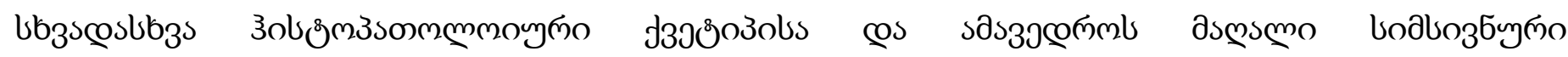

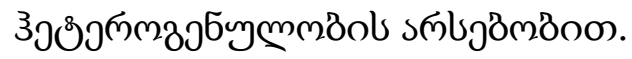

uodus

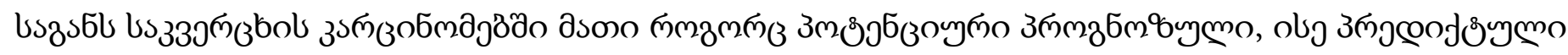

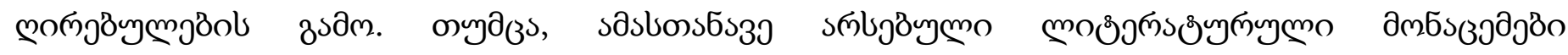

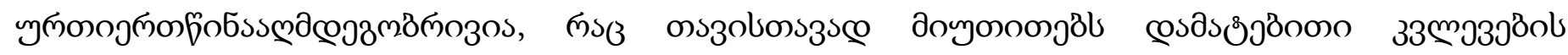

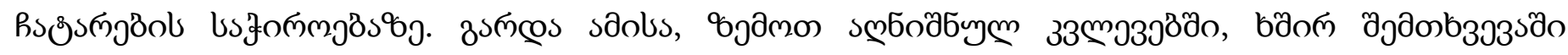

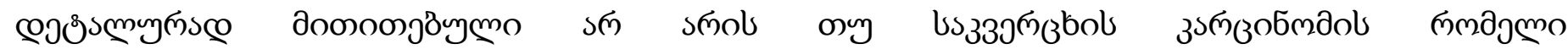

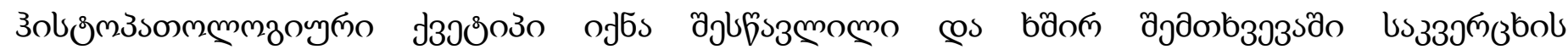

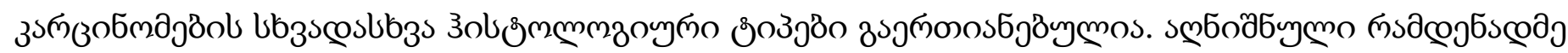

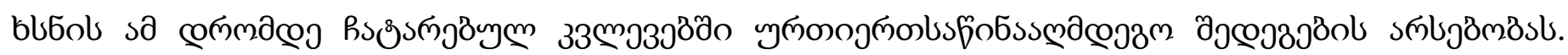

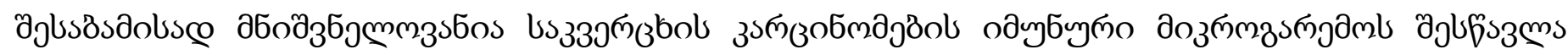

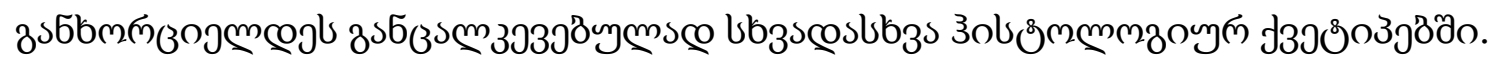




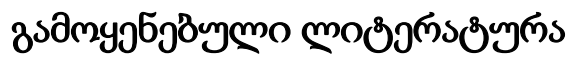

[1] Torre LA, Trabert B, DeSantis CE, et al. Ovarian cancer statistics, 2018. CA Cancer J Clin. 2018;68(4):284-296. doi:10.3322/caac.21456

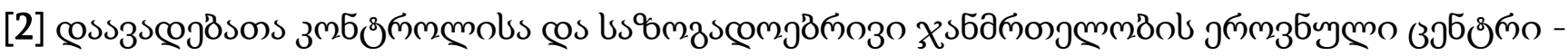

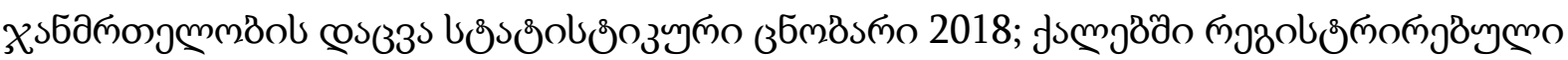

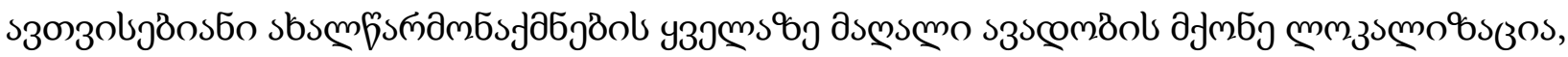

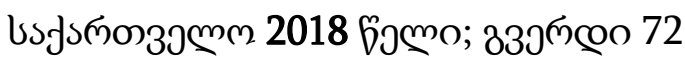

[3] Meinhold-Heerlein I, Fotopoulou C, Harter P, Kurzeder C, Mustea A, Wimberger P, Hauptmann S, Sehouli J. The new WHO classification of ovarian, fallopian tube, and primary peritoneal cancer and its clinical implications. Arch Gynecol Obstet. 2016 Apr;293(4):695-700. doi: 10.1007/s00404-016-4035-8. Epub 2016 Feb 19. Erratum in: Arch Gynecol Obstet. 2016 Jun;293(6):1367. PMID: 26894303.

[4] Paijens, S.T., Vledder, A., de Bruyn, M. et al. Tumor-infiltrating lymphocytes in the immunotherapy era. Cell Mol Immunol 18, 842-859 (2021). https://doi.org/10.1038/s41423-020-00565-9

[5] Bie, F., Tian, H., Sun, N. et al. Comprehensive analysis of PD-L1 expression, tumor-infiltrating lymphocytes, and tumor microenvironment in LUAD: differences between Asians and Caucasians. Clin Epigenet 13, 229 (2021).

[6] Santoiemma PP, Powell DJ Jr. Tumor infiltrating lymphocytes in ovarian cancer. Cancer Biol Ther. 2015;16(6):807-20. doi: 10.1080/15384047.2015.1040960. Epub 2015 Apr 20. PMID: 25894333; PMCID: PMC4622931.

[7] Yang J, Hong S, Zhang X, Liu J, Wang Y, Wang Z, Gao L, Hong L. Tumor Immune Microenvironment Related Gene-Based Model to Predict Prognosis and Response to Compounds in Ovarian Cancer. Front Oncol. 2021 Dec 13;11:807410. doi: 10.3389/fonc.2021.807410. PMID: 34966691; PMCID: PMC8710702.

[8] Xu M, Li Y, Li W, Zhao Q, Zhang Q, Le K, Huang Z, Yi P. Immune and Stroma Related Genes in Breast Cancer: A Comprehensive Analysis of Tumor Microenvironment Based on the Cancer Genome Atlas (TCGA) Database. Front Med (Lausanne). 2020 Mar 5;7:64. doi: 10.3389/fmed.2020.00064. PMID: 32195260; PMCID: PMC7066229.

[9] Lee, H.L., Jang, J.W., Lee, S.W. et al. Inflammatory cytokines and change of Th1/Th2 balance as prognostic indicators for hepatocellular carcinoma in patients treated with transarterial chemoembolization. Sci Rep 9, 3260 (2019).

[10] Gabrielson A, Wu Y, Wang H, Jiang J, Kallakury B, Gatalica Z, Reddy S, Kleiner D, Fishbein T, Johnson L, Island E, Satoskar R, Banovac F, Jha R, Kachhela J, Feng P, Zhang T, Tesfaye A, Prins P, Loffredo C, Marshall J, Weiner L, Atkins M, He AR. Intratumoral CD3 and CD8 T-cell Densities 
Associated with Relapse-Free Survival in HCC. Cancer Immunol Res. 2016 May;4(5):419-30. doi: 10.1158/2326-6066.CIR-15-0110. Epub 2016 Mar 11. PMID: 26968206; PMCID: PMC5303359.

[11] Lu, L., Barbi, J. \& Pan, F. The regulation of immune tolerance by FOXP3. Nat Rev Immunol17, 703717 (2017).

[12] Jovanović L, Janković R, Ćirković A, Jović M, Janjić T, Djuričić S, Milenković S. PD-L1 Expression in Different Segments and Histological Types of Ovarian Cancer According to Lymphocytic Infiltrate. Medicina (Kaunas). 2021 Nov 29;57(12):1309. doi: 10.3390/medicina57121309. PMID: 34946254;

PMCID: PMC8703734.

[13] Sakellariou-Thompson D, Forget MA, Hinchcliff E, et al. Potential clinical application of tumorinfiltrating lymphocyte therapy for ovarian epithelial cancer prior or post-resistance to chemotherapy. Cancer Immunol Immunother. 2019;68(11):1747-1757. doi:10.1007/s00262-019-02402-z

[14] Freedman, Ralph S., et al. "Intraperitoneal adoptive immunotherapy of ovarian carcinoma with tumor-infiltrating lymphocytes and low-dose recombinant interleukin-2: a pilot trial." Journal of immunotherapy with emphasis on tumor immunology: official journal of the Society for Biological Therapy 16.3 (1994): 198-210.

[15] Liu, J., Geng, X., Hou, J. et al. New insights into M1/M2 macrophages: key modulators in cancer progression. Cancer Cell Int 21, 389 (2021).

[16] Sun, J., Sun, J., Song, B. et al. Fucoidan inhibits CCL22 production through NF-кB pathway in M2 macrophages: a potential therapeutic strategy for cancer. Sci Rep 6, 35855 (2016).

[17] Oshi, M., Tokumaru, Y., Asaoka, M. et al. M1 Macrophage and M1/M2 ratio defined by transcriptomic signatures resemble only part of their conventional clinical characteristics in breast cancer. Sci Rep 10, 16554 (2020).

[18] Macciò, A., Gramignano, G., Cherchi, M.C. et al. Role of M1-polarized tumor-associated macrophages in the prognosis of advanced ovarian cancer patients. Sci Rep 10, 6096 (2020). 


\title{
Phenotypic features of the immune microenvironment in ovarian epithelial neoplasms and its role in tumour progression
}

\section{Critical Review}

R.Devadze ${ }^{1}$; A. Gvenetadze1; G. Burkadze²;

1. Ivane Javakhishvili Tbilisi State University;

2. Tbilisi State Medical University;

\begin{abstract}
The tumour is characterised by the presence of various amounts of lymphocytic infiltration, which is seen in different areas of cancer and has not only prognostic value the predictive value as well. Tumourinfiltrating lymphocytes (TILs) in ovarian cancer can be associated either with good prognoses or bad prognoses in some cases. The constituent which makes the tumour immune microenvironment is still under vigorous research while they may have the potential to be well modified predictive and prognostic values in ovarian cancer. The data provided by already conducted research are controversial which suggests the need for further deeper studies. Besides this fact, there is no clear determination which histological subtype of ovarian cancer is under research or in many cases all of the morphological types are united. All of these facts mentioned can clarify why the results of many pieces of research provide controversial information. It is crucial that the immune microenvironment of various morphological subtypes of ovarian carcinomas be studied separately and individually.
\end{abstract}

Keywords: Ovarian epithelial tumours; Tumour Microinvorenment (TME); Immune microenvironment; 